\title{
Algorithmic Spectral Reconstruction Using Angularly Tuned Zero-Contrast Gratings
}

\author{
Benjamin Russell ${ }^{1, *}$, Jiajun Meng ${ }^{2}$, Dandan Wen², Jasper Cadusch², Ming $\mathrm{Ye}^{2}$, and Kenneth Crozier ${ }^{1,2,3, *}$ \\ ${ }^{I}$ School of Physics, University of Melbourne, Victoria 3010, Australia \\ ${ }^{2}$ Department of Electrical and Electronic Engineering, University of Melbourne, Victoria 3010, Australia \\ ${ }^{3}$ ARC Centre of Excellence for Transformative Meta-Optical Systems (TMOS), University of Melbourne, Victoria 3010, Australia \\ *Brussell1@student.unimelb.edu.au, kenneth.crozier@unimelb.edu.au
}

\begin{abstract}
We experimentally demonstrate the algorithmic reconstruction of the infrared transmission spectrum of a polymer using a zero-contrast waveguide-grating metasurface as a filter. By changing the metasurface angle, a variety of filter functions are obtained. (C) 2020 The Author(s)
\end{abstract}

\section{Introduction}

Numerous applications exist for infrared (IR) spectroscopy, ranging from chemical identification for forensics to food quality testing and non-invasive disease diagnosis. At the time of writing, IR spectroscopy is generally carried out using systems known as Fourier transform infrared (FTIR) spectrometers. For most applications, this approach works well. There is a growing move however to the miniaturization of optical systems, to allow them to be included in platforms that include handheld consumer electronics (e.g. smartphones) and lightweight drones. This motivates the development of IR spectrometers that are small, light, low in power consumption and low in cost. One approach that has been pursued involves the use of an array of spectral filters with a matching array of detectors [1-3]. It has been shown that plasmonic nanostructures can be used to realize the filter array [1,2]. It has been shown recently however that dielectric nanostructures can support resonances with high quality factors using the bound state in the continuum (BIC) and related concepts [4]. This motivates the development of spectral filters for microspectrometers based on dielectric metasurfaces. This is the topic of this abstract. We fabricate an amorphous silicon (a-Si) on glass metasurface, of a zero-contrast grating (ZCG) design, that functions as a transmission spectral filter. An array of such filters (with different geometric parameters) could be combined with an array of infrared detectors to realize a microspectrometer. We test this approach as follows. Rather than fabricating an array of such filters, we fabricate a single filter and use it to generate multiple filter functions by changing the angle of incidence. We show that, with an algorithm, this allows us to determine (or "reconstruct") the transmission spectrum of a test sample that comprises a polymethyl methacrylate (PMMA) cuvette.

\section{Experiment}

Fabrication starts with the deposition of a-Si (1170 nm thick) onto a glass slide by plasma-enhanced chemical vapor deposition. E-beam resist (ZEP520, $400 \mathrm{~nm}$ thick) is then spin coated and exposed by e-beam lithography, to realize a line/space grating (nominal period $1100 \mathrm{~nm}$, fill factor 0.5 ). Inductively coupled plasma - reactive ion etching is then used to etch the grating to a depth of $760 \mathrm{~nm}$, followed by removal of the ZEP520 using dimethylacetamide and a solvent wash.

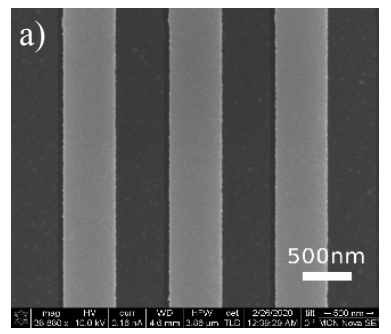

b)

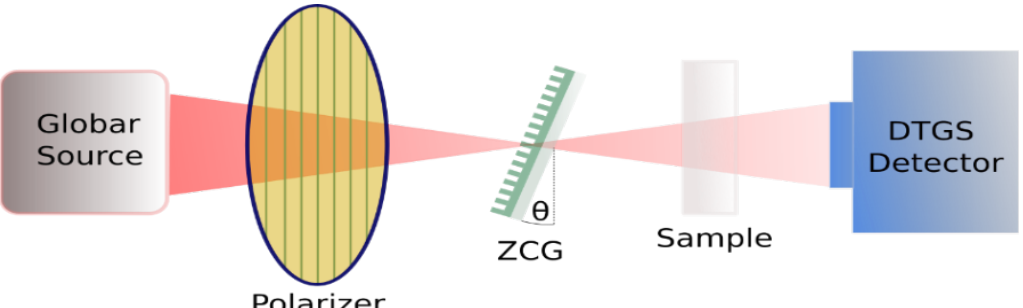

Fig 1. (a) Scanning electron micograph of a-Si metasurface (zero contrast grating, ZCG). (b) Simplified schematic of experimental set-up

An SEM image of the resultant metasurface is shown as Fig. 1a. A schematic of the experimental set-up is shown as Fig. 1b. It should be noted that this illustration is simplified in that only the infrared light source ( $\mathrm{SiC}$ globar) and the infrared detector (deuterated triglycine sulfate, DTGS) are depicted, and other parts of the system (Perkin Elmer Frontier FTIR spectrometer) such as the Michelson interferometer are not included for brevity. We first characterize our metasurface, which is mounted on a rotation stage. Note that in this step, the "sample" (of Fig. $1 b$ ) is not included. The light incident upon the metasurface has been passed through a wire grid linear polarizer, such that it is p-polarized with respect to our plane of incidence, i.e. perpendicular to the lines of the grating. 
Transmission spectra of the metasurface are measured over $M=43$ angles from $0^{\circ}$ to $42^{\circ}$ over the wavelength range $2.65 \mu \mathrm{m}-3.10 \mu \mathrm{m}(N=143$ data points). These transmission spectra are shown as Fig. 2a. We next demonstrate spectral reconstruction by determining the transmission spectrum of a sample that consists of a PMMA cuvette that is now placed into the set-up (Fig. 1b). We re-measure the transmission spectra, varying the ZCG angle as before. We then integrate each spectrum over the wavelength range $2.65 \mu \mathrm{m}-3.10 \mu \mathrm{m}$, to yield a single number. In this way, $M=43$ data points are obtained. This emulates what would be measured, were the experimental set-up to exactly correspond to the (simplified) set-up shown as Fig. 1b (with the addition of a bandpass filter). This data set is provided to an algorithm, along with the measured transmission spectra of the ZCG (without the PMMA sample). We discuss below how our algorithm then determines the spectrum of the PMMA cuvette (i.e. product of PMMA cuvette transmission spectrum and spectrum of globar light source).

The measurements produce an $M \times N$ matrix $A$ of ZCG transmission spectra, and an $M \times 1$ vector, $\boldsymbol{y}$ of transmitted power. These form the arguments of a linear equation $A \boldsymbol{x}=\boldsymbol{y}$, from which we solve for $\boldsymbol{x}$, the $N \times 1$ vector representing the transmission spectrum of the analyte. As $A$ is dimensionless and $\boldsymbol{y}$ has units of power, the reconstructed transmission will have units of power, i.e. it represents the product of the globar spectrum and the transmission spectrum of the PMMA cuvette. Least squares regression algorithms best suit problems where a sparse solution is expected, i.e. there are few non-zero elements in the solution vector. Broadband transmission spectra typically fail this criterion. We thus use a dictionary learning algorithm (DLA, e.g. [5]) to create a transformation matrix $B$ such that the problem $(A B) \boldsymbol{w}=\boldsymbol{y}$ is solved in a domain with a sparse solution vector $\boldsymbol{w}$. This can be transformed back to the wavelength domain via the relation $\boldsymbol{x}=B \boldsymbol{w}$. We also note that DLAs use minimization conditions that are similar to least squares regression algorithms. The squared differences between the vectors of a set of training spectra and their transformed sparse representations are to be minimized, while simultaneously reducing the number of non-zero terms of the sparse representation. Using a training set of 1560 assorted spectra taken from a publicly available database, we construct a dictionary that reliably provides sparse representations of the transmission spectra with signal to noise ratios exceeding 20 when transformed back to the wavelength domain. The code for the popular DLA K-SVD is taken from a publicly available DLA code repository [6] and the non-negative least squares regression algorithm is from the MathWorks linear algebra module for MATLAB. The reconstructed spectrum (of the PMMA cuvette) is shown in Fig. 2c (blue curve) along with the spectrum measured directly by our FTIR system (orange curve). It can be seen that these are in good agreement. We quantify this by the normalised root mean square deviation (NRMSD) determined by the formula $N M R S D \%=(100 / N) \times \sum_{n} \sqrt{\left(x_{n}{ }^{\prime}-x_{n}\right)^{2}} /(\max (\boldsymbol{x})-\min (\boldsymbol{x}))$ for an estimator $\boldsymbol{x}^{\prime}$. This is $8.25 \%$.
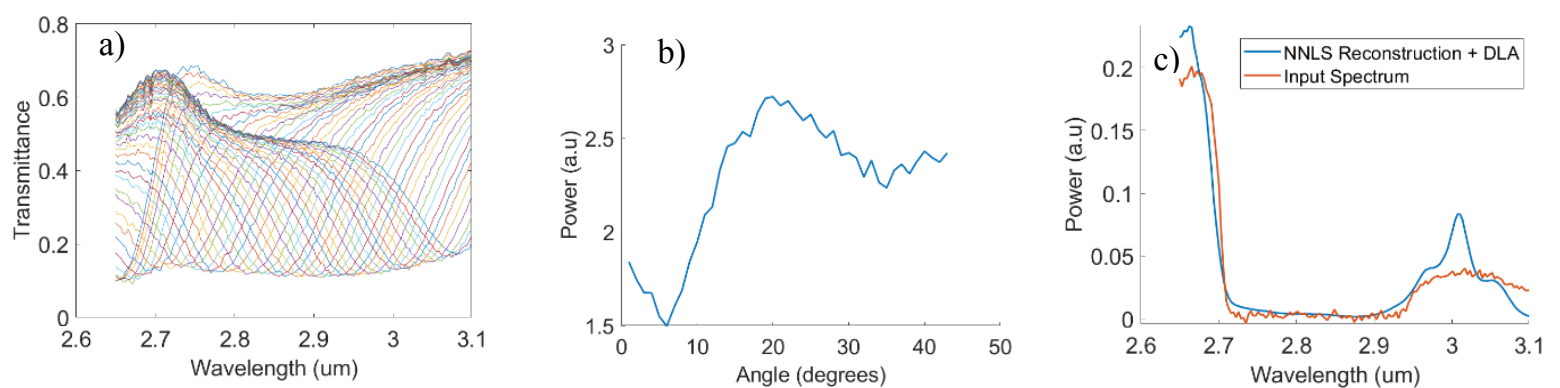

Fig 2. (a) Measured transmission spectra of the ZCG, (b) the integrated spectral power at each recorded angle and (c) Comparison between the reconstructed and measured spectra of the PMMA sample

\section{Conclusion}

We demonstrate infrared spectral reconstruction with an a-Si ZCG metasurface in the range $2.65 \mu \mathrm{m}-3.10 \mu \mathrm{m}$. This design is easily scalable to other spectral ranges by tuning the ZCG's geometric parameters proportionally.

\section{References}

[1] B.J. Craig, V.R. Shrestha, J. Meng, J.J. Cadusch, and K.B. Crozier, Experimental demonstration of infrared spectral reconstruction using plasmonic metasurfaces," Optics Letters 43, 4481-4484 (2018)

[2] B.J. Craig, J. Meng, V.R. Shrestha, J.J. Cadusch, and K.B. Crozier, "Mid- to long-wave infrared computational spectroscopy using a subwavelength coaxial aperture array," Scientific Reports 9, 13537 (2019)

[3] E.g. see Pyreos Sensor Innovation, 'A guide to choosing sensor technologies for food safety and oil analysis spectrometry applications". Downloaded from, www.pyreos.com, accessed August 7, 2019.

[4] M.V. Rybin, K.L. Koshelev, Z.F. Sadrieva, K.B. Samusev, A.A. Bodganov, M.F. Limonov, and Y.S Kivshar, "High-Q Supercavity Modes in Subwavelength Dielectric Resonators," Physical Review Letters 119, 243901 (2017)

[5] Lin Zhang et al. "A Spectral Reconstruction Algorithm of Miniature Spectrometer Based on Sparse Optimization and Dictionary Learning” Sensors 18, 644 (2018)

[6] Karl Skretting, "Dictionary Tools for Matlab", University of Stavanger, http://www.ux.uis.no/ karlsk/dle/ 


\section{University Library}

\section{- M M N E R VA A gateway to Melbourne's research publications}

Minerva Access is the Institutional Repository of The University of Melbourne

Author/s:

Russell, B;Meng, J;Wen, D;Cadusch, J;Ye, M;Crozier, K

Title:

Algorithmic Spectral Reconstruction Using Angularly Tuned Zero-Contrast Gratings

Date:

2020-01-01

Citation:

Russell, B., Meng, J., Wen, D., Cadusch, J., Ye, M. \& Crozier, K. (2020). Algorithmic Spectral Reconstruction Using Angularly Tuned Zero-Contrast Gratings. 2020 CONFERENCE ON LASERS AND ELECTRO-OPTICS PACIFIC RIM (CLEO-PR), 00, IEEE. https:// doi.org/10.1364/CLEOPR.2020.C10E_3.

Persistent Link:

http://hdl.handle.net/11343/294874 